\title{
Effects of Ageing and Hearing Thresholds on Speech Perception in Quiet and in Noise Perceived in Different Locations
}

\author{
Siti Zamratol-Mai Sarah Mukari, Nor Haniza Abdul Wahat, and Rafidah Mazlan \\ Audiology Program, School of Rehabilitation Sciences, Faculty of Health Science, Universiti Kebangsaan Malaysia, Kuala Lumpur, Malaysia
}

Received May 28, 2014

Revised September 23, 2014

Accepted October 2, 2014

\section{Address for correspondence Siti Zamratol-Mai Sarah Mukari, $\mathrm{MD}, \mathrm{MSc}, \mathrm{AuD}$ \\ Audiology Program, \\ School of Rehabilitation Sciences, Universiti Kebangsaan Malaysia, Jalan Raja Muda Abdul Aziz, 50300 Kuala Lumpur, Malaysia \\ Tel $+60-392895018$ \\ Fax $+60-326914304$ \\ E-mailzzamratol@hotmail.com}

Background and Objectives: This study investigated the effect of ageing on speech perception in quiet and in noise, with noise directed from front, right and left. Subjects and Methods: Sixty Malay native adults with normal or near normal hearing comprising of 20 young adults ( 21 to 39 years old), 20 middle aged (40 to 59 years old) and 20 older adults (60 to 74 years old) participated in this study. Their speech perception ability was measured using the Malay Hearing in Noise Test (HINT) in four test conditions; 1) in quiet (HINT Q), 2) with noise from front (HINT NF), 3) with noise from right (HINT NR), and 4) with noise from left (HINT NL). Reception thresholds for sentences (RTSs) were measured in each of the aforementioned conditions using an adaptive method. Results: The results showed that, 1) genuine age-related decline was found in speech perception performance in HINT (NF), 2) hearing threshold was a major determinant differentiating speech perception performance for HINT (Q) and HINT (NL) conditions, and 3) speech perception performance for HINT (NR) was determined by both age and hearing threshold. Conclusions: This study suggests that, in older adults, while hearing thresholds affect speech perception in quiet, other factors such as central auditory processing and cognitive functions might be more important determinant factors for speech perception performance in noise.

Korean J Audiol 2014;18(3):112-118

\section{Introduction}

For the normal hearing listener, the ability to understand speech in quiet is preserved as long as the speech is clear and audible. However, understanding speech in adverse listening conditions such as noise and reverberation is a difficult task, especially, for the elderly listeners. ${ }^{1,2)}$ Results of previous studies suggest that older listeners had poorer performance on speech understanding tasks than young listeners. ${ }^{2-5)}$ Findings from these studies suggested that age-related deficit in the peripheral auditory system, such as presbycusis, is the primary cause of speech understanding difficulties in older listeners. Hearing loss not only reduces the audibility of speech heard, but it may lead to distortion ${ }^{6)}$ to the perceived sound due to ab-

This is an Open Access article distributed under the terms of the Creative Commons Attribution Non-Commercial License (http://creativecommons. org/licenses/by-nc/3.0/) which permits unrestricted non-commercial use, distribution, and reproduction in any medium, provided the original work is properly cited. normal spectral and temporal resolution. ${ }^{1)}$ These changes impair the quality of signals received for processing by the higher level perceptual system, causing a bottom-up decline of signal processing.

Peripheral hearing loss alone, however, does not explain the speech understanding problem experienced by older adults when listening in degraded listening environments such as in noise because difficulty with speech perception may occur even in the presence of normal hearing thresholds. ${ }^{7)}$ Furthermore, speech perception is usually poorer in older adults than those in young adults with similar degree of hearing loss. ${ }^{8}$ The Working Group of the Committee on Hearing and Bioacoustics and Biomechanics of the National Research Council ${ }^{9)}$ proposed that in addition to the elevated hearing threshold, declines of central auditory system and cognitive functions also contribute to age-related deterioration in speech understanding.

Changes in central auditory processing contribute to speech perception difficulties in noise experienced by older adults. 
For example, studies using a gap detection test, a measure of temporal resolution ability have shown that older adults, independent of their hearing sensitivity, performed poorer in comparison to their younger counterparts. ${ }^{10,11)}$ Poorer temporal resolution ability among the elderly partly explains the speech perception difficulty of the older adults because good temporal resolution ability is crucial to identify phonetic contrasts $^{12)}$ as well as to help in understanding speech in background noise. $^{13)}$

Age-related cognitive changes have also been attributed to speech understanding difficulties in older listeners. ${ }^{7,14,15)}$ Cognitive processes refer to how information is acquired, stored, manipulated and used. ${ }^{16)}$ The cognitive processes that have been shown to decline with age include working memory capacity, ${ }^{17,18)}$ attention control ${ }^{19)}$ and speed of processing. ${ }^{2,14)}$ In order to correctly comprehend a sentence, listeners must be able to encode, and temporarily keep earlier heard words while simultaneously engage in the on-going processing of later words. The effect of reduced working memory capacity in speech understanding is more prominent in adverse listening conditions such as when listening in noisy environment. In such a condition, more cognitive resources are required for listening, which may reduce the working memory resources for linguistic and cognitive processing. ${ }^{20)}$ The involvement of perceptual and cognitive systems in speech perception can be considered as a multitasking system. ${ }^{20)}$ Minimal cognitive resources are required when listening to clear speech. However, when speech signals are degraded, more attention would be devoted to recover information lost in the noise. This will impair the efficiency and speed of other processes such as retrieval and storage of on-going speech, which are important in speech comprehension.

Recently, an functional MRI study has compared cortical mechanisms involved in speech perception in quiet and in noise conditions by young and older adults. ${ }^{21)}$ Their findings showed reduced activations in auditory cortex but an increased in working memory and attention-related cortical areas in older subjects, especially in noisy listening condition. Furthermore, while younger listeners displayed a more streamlined cortical network of auditory regions during speech processing in noise, older subjects showed more diffused activities involving general cognitive areas. In addition, the study also found that increased cortical activities in cognitive regions are positively correlated with behavioral speech perception performance in older adults. These findings are in line with the notion of a more intensive cognitive involvement when listening in noise than in quiet conditions. ${ }^{20)}$

Despite the abundance of research on the age-related decline on speech perception in noise, limited information is available on the effect of age on speech perception when noise is perceived in different ears. It is reasonable to speculate that in binaural listening condition, when noise is presented to the right ear, a condition, which results in more reliance on the left ear, it will be harder especially, for the older adults to perceive speech compared to when noise is presented to the left ear. That is, the effect of age on speech perception in noise is likely to be greater when noise is directed to the right ear than the left ear. This speculation is based on increasingly more difficulties focusing attention on the left ear with advancing age during dichotic listening tasks. ${ }^{22)}$

The objective of the present study was to examine the effect of age on speech perception using the Malay Hearing in Noise Test (HINT) ${ }^{23)}$ at threshold level. Specifically, the goal was to determine whether the effect of age is present in all noise conditions. In particular, this study sought to find out whether the effect of age on speech perception differs when noise is perceived from the front or either from the right or left ear. Investigation of this effect is important to provide some information of whether ageing affects speech recognition in noise differently when noise originates from different directions.

\section{Subjects and Methods}

\section{Participants}

The study involved 60 adult native Malay speakers who reported no past or present history of neurological problems. They were divided into three different age groups consisting 20 participants each: 1 ) young adults aged $21-39$ years (mean age $=$ $24.60 \pm 5.21$ years), 2) middle-aged with age ranged between $40-59$ years (mean age $=46.65 \pm 5.43$ years), and 3 ) older adults ranged in age from $60-74$ years (mean age $=64.85 \pm$ 5.21 years). All participants had bilateral normal or mild hearing loss with pure tone thresholds not greater than $25 \mathrm{~dB} \mathrm{HL}$ at octave frequencies of 250 to $2000 \mathrm{~Hz}$ and $40 \mathrm{~dB}$ or less at $4000 \mathrm{~Hz}$, and normal middle ear function with type A tympanogram. ${ }^{24)}$ All of them self-claimed to be right handed. They were recruited by means of advertisements and word of mouth. All participations were on a voluntary basis. Participants did not receive any compensation for taking part in this study.

Although, recruiting participants with matched level of hearing thresholds is ideal in a study that examines the effect of ageing on speech perception, however, this threshold-match is not easy to obtain. Sloping high-frequency sensorineural hearing loss which characterizes presbycusis is rarely found in young individuals. ${ }^{6}$ Therefore, finding younger individuals with such a configuration is difficult. ${ }^{5,25)}$ Similarly, it is very difficult to find older adults with normal hearing as seen in younger subjects. As elevation of auditory threshold in older 
adults is likely to affect speech perception performance, ${ }^{5)}$ in this study the potential effects of hearing loss are controlled through performing, for each measure, analysis of covariance, with the four frequency $(0.5,1,2$, and $4 \mathrm{kHz})$ average hearing threshold as the covariate. Previous studies have also used similar method to control the possible effect of hearing threshold on the results. ${ }^{5,26)}$

\section{Stimuli}

The Malay HINT, ${ }^{23)}$ an adaptive open-set speech recognition test, was used to determine the reception threshold for sentences (RTSs). The HINT was delivered using HINT hardware routed through Pro 7.0 Audiometric System (Bio-Logic Systems Corp, Mundelein, IL, USA) coupled to a computer desk top and supra-aural TDH 39 headphones. HINT is equipped with audio sound processing that simulates the source locations for the speech $\left(0^{\circ}\right)$ and noise $\left(0^{\circ}, 90^{\circ}\right.$, and $\left.270^{\circ}\right)$ azimuths by processing the speech and noise materials with digital filters prior to headphones presentations. ${ }^{27)}$ The Malay HINT consists of 12 phonemically balanced lists of 20 short Malay sentences spoken by a male native Malay speaker. Each sentence has between four to six words and is graded at first grade reading level. The noise used in HINT is steady state, which matched the average long-term spectrum of the sentences. During testing, the sentences were only played once, and the subjects were encouraged to repeat the complete sentences and also to guess if they were unsure of the sentences heard. Sentences were scored as correct if the listener repeated all the words correctly.

\section{Procedure}

The HINT was conducted in a single walled sound treated booth using headphones in four different conditions: in quiet (HINT Q), noise perceived from front (NF), noise perceived from right (NR), and noise perceived from left (NL). In all test conditions, speech was presented from the front. The participant was instructed to repeat each of the sentences presented. The tester scored the sentence as correct if the participant repeated the whole sentence correctly. HINT was conducted using the adaptive technique whereby the intensity of the speech signal was adjusted according to the subject's responses. One list of 20 sentences each was used to determine the reception threshold for sentences (RTS) for each HINT conditions. For HINT (Q), the first sentence was presented at the listener's three frequencies average hearing thresholds $(0.5,1$, and $2 \mathrm{kHz})$. If the sentence was not repeated correctly the sentence was presented again, and the intensity was raised by $4 \mathrm{~dB}$ increments until the listener heard it correctly. Subsequence sentences were presented once each. For sentences 2 to 4 , the intensity was increased following incorrect sentence repetition or lowered by $4 \mathrm{~dB}$ if sentence was correctly repeated. For sentences 5 through 20 , the level was varied in 2-dB steps depending on the listener's response. RTS was calculated based on the average presentation level of the 5th through the 20th sentences and the level at which the 21 st sentence would be presented.

For the measurement of RTS for HINT in noise conditions, noise level was fixed at $65 \mathrm{dBA}$, while speech intensity was raised or decreased depending on the listener's response. The first sentence was presented at $61 \mathrm{dBA}$ for HINT (NF), and at $55 \mathrm{dBA}$ for the other two HINT in noise conditions. The presentation steps during testing were automated by the Malay HINT software.

The order of presentation of the HINT conditions was randomized across participants. All participants were tested in a single test session. The procedure, including the preliminary tests, took about 1 hour.

The experimental protocol and procedures in this study were approved by the Universiti Kebangsaan Malaysia Research Ethics Committee (approval number NN-038-2013).

\section{Data analysis}

For each HINT condition, a one way analysis of variance (ANOVA) was conducted to find out the effect of age on speech perception ability. Since there was a trend of poorer hearing threshold with age, it is possible that the significant differences between age groups revealed by the ANOVA analyses was attributable to poorer hearing thresholds seen in the older age groups. Therefore, to examine the effect of hearing thresholds and age group on RTSs, an analysis of covariate (ANCOVA) was employed. In this analysis RTS was used as a dependent variable, age group as a fixed factor and the mean four frequency average (FFA) hearing threshold as the covariate.

\section{Results}

\section{Hearing thresholds}

Sixty participants consisting of 20 subjects in each age group enrolled in this study. Table 1 shows the mean ages, FFA hearing threshold for the right and left ears and standard deviations (SDs) for each group. There was a trend for age-related decline in hearing thresholds. A one-way ANOVA revealed significant differences between age groups $[\mathrm{F}(2,57)=30.88, p<0.001]$. Scheffe posthoc analysis indicated that the groups' FFA hearing thresholds significantly differed from each other $(p<0.01)$.

\section{The effect of age on RTS for HINT in quiet}

One way ANOVA analysis with RTS as a dependent variable and age group and the independent variable show a significant effect of age $[\mathrm{F}(2,57)=16.08, p<0.001]$. A one-way 
Table 1. Means age, right four frequency average (FFA) $(0.5,1,2$, and $4 \mathrm{kHz}$ ) and standard deviations (SDs) of each groups

\begin{tabular}{lccc}
\hline Age group & $\begin{array}{c}\text { Mean age } \\
\text { (years) }(\mathrm{SD})\end{array}$ & $\begin{array}{c}\text { Mean right } \\
\text { FFA (dB) (SD) }\end{array}$ & $\begin{array}{c}\text { Mean left } \\
\text { FFA (dB) (SD) }\end{array}$ \\
\hline Young & $24.6(5.2)$ & $12.2(5.6)$ & $9.9(4.3)$ \\
Middle-aged & $46.7(5.4)$ & $15.6(3.9)$ & $19.9(3.6)$ \\
Older adults & $64.9(5.2)$ & $23.0(7.1)$ & $21.9(6.2)$ \\
\hline
\end{tabular}

ANCOVA with average hearing threshold as a covariate was conducted to control the effect of hearing threshold. The result revealed a significant effect of hearing thresholds on the RTS measured in quiet condition, signifying that higher mean FFA hearing threshold was related to higher RTS $[F(1,56)=32.69$, $p<0.001]$. When the effect of hearing threshold was controlled, no significant effect of age group on RTS $(p>0.05)$ was found, indicating that RTS for HINT in quiet was related to hearing threshold but not to age group.

\section{The effect of age and direction of noise on RTS}

The differential effects of age and hearing threshold on RTSs for HINT in noise differ depending on the noise location. For HINT (NF) condition, the effect of age group was significant $[F(2,56)=7.22, p<0.01]$, while hearing threshold had no significant effect on RTS. Pair-wise comparisons revealed that the older adults group had significantly poorer RTS than both the middle aged $[\mathrm{t}(56)=3.61, p<0.001]$, and young adults [ $\mathrm{t}$ $(56)=3.24, p<0.01]$. There was no significant difference between the mean RTSs of the two younger groups ( $p>0.05)$, signifying that performance in noise front dropped significantly after 60 years old.

The one-way ANOVA to assess the effect of age group on the RTS for HINT (NR) revealed a significant effect of age [F $(2,52)=17.89, p<0.001]$. ANCOVA with the average hearing threshold as a covariate revealed that the RTS for HINT (NR) was significantly influenced by both hearing threshold and age group. Poorer hearing threshold was associated with higher RTS $[F(1,56)=4.88, p<0.05]$. The effect of age group persisted, even after controlling the effect of hearing threshold $[\mathrm{F}$ $(2,56)=3.62, p<0.05]$. Pair-wise comparisons showed that the older adults group had significantly poorer adjusted mean RTS than young adults [ $\mathrm{t}(56)=2.69, p<0.01]$, but no significant difference was observed between the adjusted mean RTSs of the older adults and the middle-aged groups. Neither was there significant difference between the adjusted mean RTSs of the young and middle aged groups, suggesting that difficulty listening with NR significantly declined after 60 years old.

For HINT (NL), one way ANOVA with age group as an independent variable revealed a significant main effect of age $[\mathrm{F}$ $(2,57)=7.11, p<0.01]$. ANCOVA with the average hearing threshold as a covariate showed that the RTS for HINT (NL)

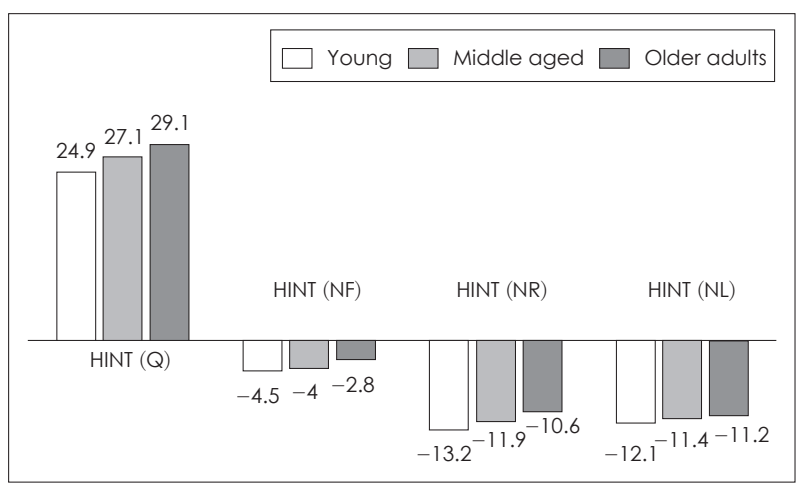

Fig. 1. Adjusted mean reception threshold for sentences of HINT $(\mathrm{Q})$ in $\mathrm{dB}$, HINT (NF), HINT (NR) and HINT (NL) in signal to noise ratio across age groups.

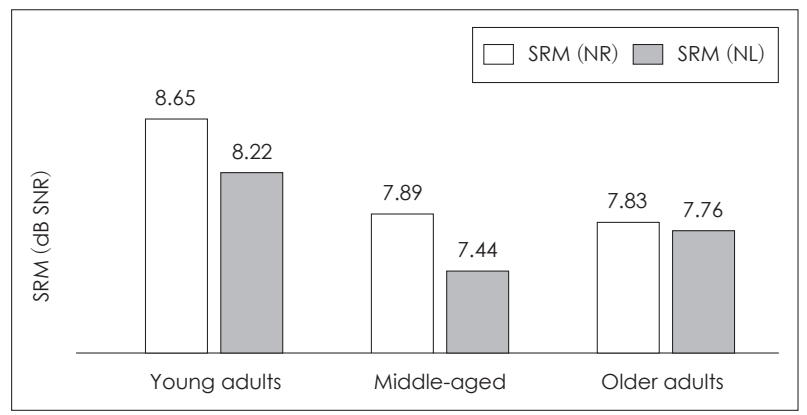

Fig. 2. Means spatial release from masking (SRM) achieved in noise right (NR) and noise left (NL) conditions in young adults, middleaged and older adults groups.

condition was significantly influenced by hearing threshold $[\mathrm{F}(1,56)=4.18, p<0.05]$, implying higher hearing threshold was associated with higher RTS. The effect of age group disappeared after controlling the effect of hearing threshold, signifying that RTS (NL) was mostly influenced by hearing threshold rather than age. Fig. 1 summarizes the adjusted mean values of RTS for HINT(Q), HINT (NF), HINT (R), and HINT (L), with the mean FFA hearing thresholds factored out.

\section{Spatial release from masking (SRM)}

The magnitudes of the spatial release from masking (SRM) in HINT (NR) and HINT (NL) conditions were calculated by subtracting the RTSs in noise right and noise left from RTS in noise front, respectively (e.g., SRM noise right=RTS noise frontRTS noise right). Fig. 2 shows the SRM achieved in noise right and noise left conditions, across age groups. A mixed design ANOVA revealed neither significant effect of noise perceived location nor age group $(p>0.05)$, indicating that the magnitudes of SRM were similar across noise perceived location and age groups. In addition, there was no significant correlation between the mean FFA hearing threshold and SRM values, which suggests that hearing loss did not impair the benefit from spatial separation of noise. 


\section{Discussion}

The main aim of this study was to examine the age-related performance difference in RTSs obtained using adaptive method by mean of HINT (Q) and in three noise conditions, i.e., NF, $\mathrm{NR}$, and NL, by controlling the hearing thresholds.

The first question addressed in this study was whether the RTSs in quiet and in noise by the young, middle-aged and older adults were different from each other. There was a general trend for increased in RTSs with increasing age in all HINT conditions. Interestingly, however, the effect of age remained in only some of these conditions when the mean FFA hearing threshold was factored out. For HINT in quiet, when the three groups were equated for mean FFA hearing threshold, their RTSs did not differ significantly. This signifies that the worsening of RTS with increasing age was associated to impairment of the peripheral hearing loss experienced by the older age groups. This finding is expected because listening to simple sentences such as those used in the present study, in quiet does not overtax the cognitive function. Therefore, the performance largely depends on the clarity of the signals at input lev$\mathrm{el}^{20)}$ This finding lends further support to earlier investigation which found that peripheral hearing loss contributed to reduce speech reception threshold in quiet. ${ }^{24)}$

In contrast, for HINT (NF), a robust effect of age group on RTS was found even after hearing threshold was factored out. The older adults group, regardless of hearing thresholds, required approximately $2 \mathrm{~dB}$ greater signal to noise (SNR) to recognize $50 \%$ of the sentences than both the young and middleaged groups, when both speech and noise were perceived from the front. Given that the means RTS of the young and middleaged groups did not differ significantly from each other and that both differed from the older adults group, suggest that age related decline in listening in noise is more noticeable after 60 years old. This finding suggests that the decrement in speech perception in noise found in the older adults group is attributable by factors other than hearing thresholds, such as the decline in cognitive and central auditory processing functions associated with ageing. ${ }^{27,15,28)}$ Unlike listening to speech signals in quiet, which largely depends on bottom-up processing, listening to speech in the presence of background noise heavily relies on cognitive function. ${ }^{7)}$ This is because listening to degraded speech, such as when listening in poor SNRs, increases demand on cognitive functions such as attention and working memory to make up for the lost in acoustic information and perceptual processing. ${ }^{20)}$ Thus, ageing which is known to be associated with a decline in cognitive function and auditory processing is likely to affect listening performance in this condition. However, the contribution of cognitive and central au- ditory deficits to the difficulty with speech perception in noise among older adults in this study cannot be ascertained as there was no auditory processing or cognitive tests included.

Although the majority of previous studies on spatial processing suggest that SRM declines with age, there are other studies which find otherwise..$^{29)}$ Dubno, et al. ${ }^{30)}$ who studied spatial processing in young adults with normal hearing, old adults with normal hearing and old adults with hearing impairment found that both aging and hearing impairment reduce spatial processing ability. A more recent study investigated the effects of hearing impairment and aging on spatial processing in a group of participants aged from seven to 89 years and hearing thresholds ranging from normal to moderately severe hearing loss. Their results revealed that spatial processing is significantly affected by hearing impairment but not by aging. ${ }^{30)}$ The present study in that aging is not related to spatialprocessing ability partially concurs with the findings of Glyde, et al. ${ }^{31)}$ The lack of significant effect of hearing level on spatial processing found in this study could be due to differences in methodology and or participants' hearing level. The fact that all age groups in the present study benefited similarly from perceived spatial separation further supports that poorer speech perception in HINT (NF) observed in the older adults group is attributed by poorer processing and cognitive function rather than poorer hearing thresholds.

Both FFA hearing threshold and age had significant effects on RTS for HINT (NR). These findings suggest that RTS for HINT (NR) is affected by hearing loss and age acting in tandem and that poorer RTS in this listening condition observed in older adults is more than the simple consequence of hearing threshold elevations. In HINT (NR) condition, spectrally matched noise was presented at $65 \mathrm{dBA}$ and perceived in the right ear while sentences were presented at a relatively lower level at a simulated $0^{\circ}$ azimuth (at the front). This condition generally produced a perceptually greater SNR and "cleaner signal" in the left than the right ear. Although the neural pathways from the two ears project to both auditory cortices, the input from the contralateral ear produces a stronger representation in the auditory cortex and could suppress information from the ipsilateral ear. ${ }^{32)}$ It is also an established fact that in most people left hemisphere is dominant for language processing. ${ }^{33)}$ Based on these premises, in HINT (NR), sentences heard from the left ear are mainly routed to the right hemisphere before they are transferred through the corpus callosum to the left hemisphere to be processed, whereas noise delivered to the right ear is routed to the left hemisphere. Age-related degenerative changes of corpus callosum seen in older adults ${ }^{34)}$ may result in loss of information when competing signals are present. Poor signal quality that reaches the left hemisphere 
causes a low signal-to-noise ratio that may degrade signal processing. Partly because of the decrease in hearing sensitivity, the clarity of acoustic signal throughout the auditory system is expected to be degraded in the older listeners. It is suggested that this degradation in the signal clarity contributes to the speech perception problems in noise reported by many older adults and as observed in the present study. Furthermore, from the top-down view, perception of degraded speech such as that in low SNR is also influenced by cognitive factors such as selective attention, speed of processing and working memory that are known to decline with age. ${ }^{15,17,35)}$

It is interesting that in comparison to HINT (NR), the RTS for HINT (NL) was affected by FFA hearing threshold but not by age group. This implies the reliance of HINT (NL) on bottom-up rather than top-down processing. Given that the contralateral pathways from the ears to the auditory cortices are more prominent than the ipsilateral routes and the left cerebral dominance in language processing HINT (NL) provides a more conducive listening than HINT (NR). First, the sentences that are presented to the right ear will be directly routed to the left hemisphere, which is dominant for language processing, hence better quality signals are made available for processing. Second, processing of sentences by the left hemisphere is easier because most of the noise is routed to the right hemisphere, thus producing a better SNR in the left hemisphere. The relatively more favorable listening provided in HINT (NL) condition resulting from the efficient bottom-up processing may minimize the reliance on the top-bottom processing, and hence explain the lack of age effect on the RTS. This explanation is supported by previous studies, which consistently showed that the effect of ageing significantly impaired the performance of the left ear during dichotic listening, while the right ear performance remains relatively unaffected. ${ }^{18,28)}$

Overall the results of the present study agree with that of Divenyi and Haupt ${ }^{25)}$ in that speech understanding deficits in older adults can be classified into two categories. The first category encompasses auditory measures that exhibit a different between older adults and young subjects only when the effect of hearing level is present. The second comprises measures exhibiting a robust age effect even after the effect of hearing sensitivity is factored out. The present study suggests that the deterioration in performance observed in older age groups under HINT (Q) and HINT (NL) was primarily due to a declined in the peripheral hearing thresholds. On the other hand the performance deficits in HINT (NF) and possibly (HINT NR) conditions were mainly due to a decline of processes, other than hearing acuity, associated with ageing.

\section{Conclusion}

Taken together with the results of previous findings, ${ }^{25)}$ the clinical implications of the present study is that listening with noise from front or possibly from the direction of the right ear might requires greater cognitive loading compared to when noise is coming from the left side. Subsequently, older individuals might have greater difficulty listening with noise coming from the right than from the left ear.

Despite of the statistical significance of the effect of age on HINT (NR), however, it is important to note the present study did not include any cognitive or central auditory test battery. Future studies should examine cognitive measures such as working memory capacity and attention as well as auditory processing tests to assess the relationships and interactions between those variables as explanatory factors to the decline in speech perception performance in noise in older adults. In the present study all participants in the young adults group had normal hearing, and the effects of aging and hearing loss were investigated using hearing level as a covariate. A better method would be to also include young hearing-impaired listeners as participants.

\section{Acknowledgments}

The study was funded by the Universiti Kebangsaan Malaysia Research Grant, UKM-GUP-2011-127.

The authors acknowledge the contribution from Siti Nadia Zainal and Umi Asura Sanusi who were involved in the data collection.

\section{REFERENCES}

1) Humes LE. Speech understanding in the elderly. J Am Acad Audiol 1996;7:161-7.

2) Gordon-Salant $S$. Hearing loss and aging: new research findings and clinical implications. J Rehabil Res Dev 2005;42(4 Suppl 2):9-24.

3) Dubno JR, Dirks DD, Morgan DE. Effects of age and mild hearing loss on speech recognition in noise. J Acoust Soc Am 1984;76:87-96.

4) van Rooij JC, Plomp R, Orlebeke JF. Auditive and cognitive factors in speech perception by elderly listeners. I: Development of test battery. J Acoust Soc Am 1989;86:1294-309.

5) Divenyi PL, Stark PB, Haupt KM. Decline of speech understanding and auditory thresholds in the elderly. J Acoust Soc Am 2005;118:1089100.

6) Plomp R. Auditory handicap of hearing impairment and the limited benefit of hearing aids. J Acoust Soc Am 1978;63:533-49.

7) Pichora-Fuller MK. Cognitive aging and auditory information processing. Int J Audiol 2003;42 Suppl 2:2S26-32.

8) Gelfand SA, Piper N, Silman S. Consonant recognition in quiet and in noise with aging among normal hearing listeners. J Acoust Soc Am 1986;80:1589-98.

9) Speech understanding and aging. Working Group on Speech Understanding and Aging. Committee on Hearing, Bioacoustics, and Biomechanics, Commission on Behavioral and Social Sciences and Education, National Research Council. J Acoust Soc Am 1988;83:85995.

10) Goŕdon-Salant S, Fitzgibbons PJ. Temporal factors and speech recognition performance in young and elderly listeners. J Speech Hear 
Res 1993;36:1276-85.

11) Snell KB. Age-related changes in temporal gap detection. J Acoust Soc Am 1997;101:2214-20.

12) Dorman MF, Marton K, Hannley MT, Lindholm JM. Phonetic identification by elderly normal and hearing-impaired listeners. J Acoust Soc Am 1985;77:664-70.

13) Feng Y, Yin S, Kiefte M, Wang J. Temporal resolution in regions of normal hearing and speech perception in noise for adults with sloping high-frequency hearing loss. Ear Hear 2010;31:115-25.

14) Salthouse TA. The processing-speed theory of adult age differences in cognition. Psychol Rev 1996;103:403-28.

15) Gordon-Salant S, Fitzgibbons PJ. Selected cognitive factors and speech recognition performance among young and elderly listeners. J Speech Lang Hear Res 1997;40:423-31.

16) Rabbitt P. An age-decrement in the ability to ignore irrelevant information. J Gerontol 1965;20:233-8.

17) Humes LE, Floyd SS. Measures of working memory, sequence learning, and speech recognition in the elderly. J Speech Lang Hear Res 2005;48:224-35.

18) Mukari SZ, Umat C, Othman NI. Effects of age and working memory capacity on pitch pattern sequence test and dichotic listening. Audiol Neurootol 2010;15:303-10.

19) Passow S, Westerhausen R, Wartenburger I, Hugdahl K, Heekeren $\mathrm{HR}$, Lindenberger $\mathrm{U}$, et al. Human aging compromises attentional control of auditory perception. Psychol Aging 2012;27:99-105.

20) Pichora-Fuller MK, Schneider BA, Daneman M. How young and old adults listen to and remember speech in noise. J Acoust Soc Am 1995; 97:593-608.

21) Wong PC, Jin JX, Gunasekera GM, Abel R, Lee ER, Dhar S. Aging and cortical mechanisms of speech perception in noise. Neuropsychologia 2009;47:693-703.

22) Bouma A, Gootjes L. Effects of attention on dichotic listening in elderly and patients with dementia of the Alzheimer type. Brain Cogn 2011;76:286-93.
23) Quar TK, Mukari SZ, Abdul Wahab NA, Abdul Razak R, Omar M, Maamor N. The Malay hearing in noise test. Int J Audiol 2008;47:37980.

24) Frisina DR, Frisina RD. Speech recognition in noise and presbycusis: relations to possible neural mechanisms. Hear Res 1997;106:95104.

25) Divenyi PL, Haupt KM. Audiological correlates of speech understanding deficits in elderly listeners with mild-to-moderate hearing loss. I. Age and lateral asymmetry effects. Ear Hear 1997;18:42-61.

26) McCoy C, Butler M, Broekhoff J. Effects of age and sex on dichotic listening: the SSW test. J Aud Res 1977;17:263-8.

27) Soli SD, Wong LL. Assessment of speech intelligibility in noise with the Hearing in Noise Test. Int J Audiol 2008;47:356-61.

28) Jerger J, Chmiel R, Allen J, Wilson A. Effects of age and gender on dichotic sentence identification. Ear Hear 1994;15:274-86.

29) Glyde H, Hickson L, Cameron S, Dillon H. Problems hearing in noise in older adults: a review of spatial processing disorder. Trends Amplif 2011;15:116-26.

30) Dubno JR, Ahlstrom JB, Horwitz AR. Spectral contributions to the benefit from spatial separation of speech and noise. J Speech Lang Hear Res 2002;45:1297-310.

31) Glyde H, Cameron S, Dillon H, Hickson L, Seeto M. The effects of hearing impairment and aging on spatial processing. Ear Hear 2013; 34:15-28.

32) Kimura D. Functional asymmetry of the brain in dichotic listening. Cortex 1967;3:163-78.

33) Knecht S, Dräger B, Deppe M, Bobe L, Lohmann H, Flöel A, et al. Handedness and hemispheric language dominance in healthy humans. Brain 2000;123 Pt 12:2512-8.

34) Greenwald RR, Jerger J. Aging affects hemispheric asymmetry on a competing speech task. J Am Acad Audiol 2001;12:167-73.

35) de Fockert JW. Keeping priorities: the role of working memory and selective attention in cognitive aging. Sci Aging Knowledge Environ 2005;2005:pe34. 